\title{
Plottable real number functions and the computable graph theorem
}

\author{
Vasco Brattka \\ Theoretische Informatik I, FernUniversität in Hagen, D-58084 Hagen, Germany \\ E-Mail: vasco.brattka@fernuni-hagen.de
}

\begin{abstract}
The Graph Theorem of classical recursion theory states that a total function on the natural numbers is computable, if and only if its graph is recursive. It is known that this result can be generalized to real number functions where it has an important practical interpretation: the total computable real number functions are precisely those which can be effectively plotted with any given resolution. We generalize the Graph Theorem to appropriate partial real number functions and even further to functions defined on certain computable metric spaces. Besides the non-uniform version of the Graph Theorem which logically relates computability properties of the function and computability properties of its graph, we also discuss the uniform version: given a program of a function, can we algorithmically derive a description of its graph? And, vice versa, given a description of the graph, can we derive a program of the function? While the passage from functions to graphs always is computable, the inverse direction from graphs to functions is problematic and it turns out that the answers to the uniform and the non-uniform questions do not coincide. We prove that in both cases certain topological and computational properties (such as compactness or effective local connectedness) are sufficient for a positive answer and we provide counterexamples which show that the corresponding properties are not superfluous. Additionally, we briefly discuss the special situation of the linear case.
\end{abstract}

Keywords: Computable real number functions, recursive graphs

\section{Introduction}

The Graph Theorem of classical recursion theory states that a total function $f: \mathbb{N} \rightarrow \mathbb{N}$ on the natural numbers $\mathbb{N}:=\{0,1,2, \ldots\}$ is computable, if and only if its graph, i.e. the set $\operatorname{graph}(f):=\{(n, f(n)): n \in \mathbb{N}\} \subseteq \mathbb{N} \times \mathbb{N}$, is recursive [15]. In particular, this result shows that the notions of a computable function and of a recursive subset are logically of the same power (in the sense that one could be derived from the other). 
We will investigate generalizations of the Graph Theorem from the point of view of computable analysis which is the Turing machine based theory of computability on real numbers and other topological spaces. Pioneering work on this theory has been presented by Turing [17], Banach and Mazur [1], Lacombe [13] and Grzegorczyk [11]. Recent monographs have been published by Pour-El and Richards [16], Ko [12] and Weihrauch [18].

Roughly speaking, a real number function $f: \mathbb{R}^{n} \rightarrow \mathbb{R}$ is computable in the sense of computable analysis, if and only if there exists a Turing machine which transfers better and better rational approximations of $x$ into corresponding approximations of $f(x)$. Based on the well-known physical Church-Turing Thesis [15] one might derive a thesis which underlines that this notion has a direct relation to the abilities of physical computers:

Thesis 1.1 A function $f: \mathbb{R}^{n} \rightarrow \mathbb{R}$ is computable, if and only if it can be evaluated on a physical computer with arbitrary given precision.

It is an important feature ${ }^{1}$ of computable analysis that the Graph Theorem can be generalized to continuous real number functions $f: \mathbb{R} \rightarrow \mathbb{R}$ (see [18], this fact has first been proved for continuous functions $f:[0,1] \rightarrow \mathbb{R}$ by Zhou [21]). Thus, a continuous function $f: \mathbb{R} \rightarrow \mathbb{R}$ is computable, if and only if $\operatorname{graph}(f):=\{(x, f(x)): x \in \mathbb{R}\} \subseteq \mathbb{R} \times \mathbb{R}$ is recursive.

Here, a closed subset $A \subseteq \mathbb{R} \times \mathbb{R}$ is called recursive, if and only if its distance function $d_{A}: \mathbb{R} \times \mathbb{R} \rightarrow \mathbb{R},(x, y) \mapsto \inf _{(a, b) \in A}\|(x, y)-(a, b)\|$ is computable (where $\|(x, y)\|:=$ $\max \{|x|,|y|\}$ denotes the maximum norm). Similarly as the computable functions, the recursive subsets have a very practical meaning: these are exactly those subsets which can be effectively displayed.

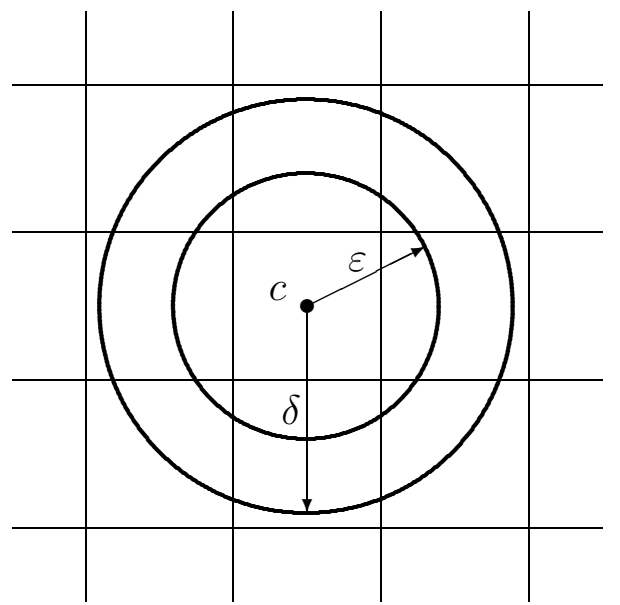

Fig. 1. Determination of a pixel color.

This is easy to see, since given a screen with pixels of a certain resolution, the color of any given pixel can be determined by computing the distance $d_{A}(c)$ of the center $c$ of the

$\overline{1}$ This feature is, for instance, not shared by the so-called BSS-model where it only holds for algebraically closed fields [10] 
pixel to the set $A$ (cf. the discussions in $[8,18]$ ). If $\varepsilon$ is the radius of a ball with center $c$ which completely contains the pixel and if $\delta>\varepsilon$ is the radius of such a ball which is completely contained in the neighbour pixels, then $d_{A}(c)<\delta$ could lead to a black pixel and $d_{A}(c)>\varepsilon$ to a white pixel (depending on which inequality can be verified first, see Figure 1). Since in principle the decision cannot be made with infinite precision, there will always remain a certain area of indetermination (in our case the values in the interval $\varepsilon<d_{A}(c)<\delta$ ) where the pixel might be white as well as black. However, this procedure guarantees that, on the one hand, the pixel will turn black if the set hits the pixel and if the pixel is turned to black, then the set does at least hit one of the neighbour pixels (or the pixel itself). Altogether, this justifies to derive the following thesis.

Thesis 1.2 A closed subset $A \subseteq \mathbb{R}^{2}$ is recursive, if and only if it can be displayed by $a$ physical computer with arbitrary given resolution.

If we apply these considerations to functions $f: \mathbb{R} \rightarrow \mathbb{R}$ with a recursive graph, then we obtain that these functions are plottable in the sense that there exists an algorithm which can display any part of the graph with any given resolution. Thus, in particular any computable function $f$ is plottable.

Unfortunately, this pleasant property does not hold true for partial functions. Topologically, $\operatorname{graph}(f):=\{(x, f(x)): x \in \operatorname{dom}(f)\} \subseteq \mathbb{R} \times \mathbb{R}$ is not even closed in general, if $f: \subseteq \mathbb{R} \rightarrow \mathbb{R}$ is a partial computable function. And even if the graph is closed, it is not necessarily recursive, as the following counterexample shows.

Example 1.3 There exists a partial computable function $f: \subseteq \mathbb{R} \rightarrow \mathbb{R}$ with $\operatorname{dom}(f)=$ $\mathbb{R} \backslash \mathbb{N}$ such that graph $(f) \subseteq \mathbb{R} \times \mathbb{R}$ is closed but not recursive closed.

Proof. Let $a: \mathbb{N} \rightarrow \mathbb{N}$ be some computable and injective enumeration of a non-recursive set $K=\operatorname{range}(a)$. Let the partial function $t: \subseteq \mathbb{R} \rightarrow \mathbb{R}$ be defined by $t(x):=\tan \left(\pi\left(x-\frac{1}{2}\right)\right)$. Then $t$ is a computable function with $\operatorname{dom}(t)=\mathbb{R} \backslash \mathbb{N}$. We will define $f: \subseteq \mathbb{R} \rightarrow \mathbb{R}$ by modifying $t$. Therefore, for any $n \in K=\operatorname{range}(a)$ let $p_{n}:\left[n+2^{-a^{-1}(n)-4}, n+2^{-a^{-1}(n)-2}\right] \rightarrow \mathbb{R}$ be the polygon defined by the vertices

$$
\left(n+2^{-a^{-1}(n)-4}, t\left(n+2^{-a^{-1}(n)-4}\right)\right),\left(n+2^{-a^{-1}(n)-3}, 0\right),\left(n+2^{-a^{-1}(n)-2}, t\left(n+2^{-a^{-1}(n)-2}\right)\right) .
$$

Now we define $f: \subseteq \mathbb{R} \rightarrow \mathbb{R}$ by

$$
f(x):=\left\{\begin{array}{l}
p_{n}(x) \text { if }(\exists n \in K) x \in\left[n+2^{-a^{-1}(n)-4}, n+2^{-a^{-1}(n)-2}\right] \\
t(x) \text { else }
\end{array}\right.
$$

for all $x \in \mathbb{R}$. Then $f$ with $\operatorname{dom}(f)=\mathbb{R} \backslash \mathbb{N}$ is computable, since $a$ is computable. On the other hand, graph $(f) \subseteq \mathbb{R} \times \mathbb{R}$ is obviously closed but not recursive closed, since $K=\operatorname{range}(a)$ is not co-r.e. and

$$
n \notin K \Longleftrightarrow d_{\operatorname{graph}(f)}\left(n+\frac{1}{8}, 0\right)>\frac{1}{8} .
$$


The intuitive reason for this inconsistency between computability of partial functions and plottability of their graphs is the following: the computation of a function might take longer and longer when we approach the singularities while the decision for any pixel in a plot of its graph has to be made after finite time. In other words: the topology of the display does not take care of the singularities. It is worth mentioning that such pathologies are well-known in practice. The following picture shows a standard plot of the tangent function (produced by gnuplot 3.7.3 with default options):

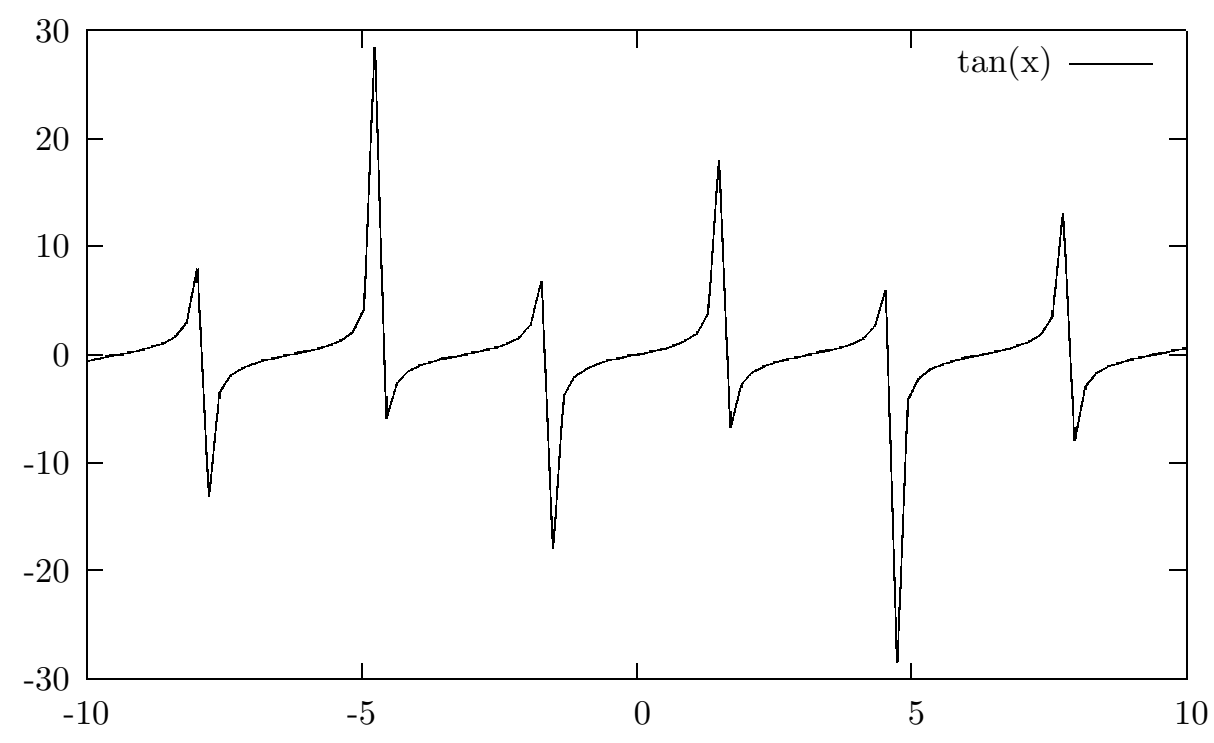

Fig. 2. An incorrect plot of the tangent function.

However, one might overcome this deficiency by being a bit more flexible in the definition of recursiveness. With respect to the Graph Theorem it seems to be much more natural to consider partial functions $f: \subseteq \mathbb{R} \rightarrow \mathbb{R}$ as total functions $f: X \rightarrow \mathbb{R}$ with $X=$ $\operatorname{dom}(f)$ and their graphs graph $(f)$ as subsets of $X \times \mathbb{R}$. In case of our example above this means changing the topology of the display in the following way: when we approach the singularities the resolution has to be increased correspondingly.

Actually, we will even address a slightly more general question in the following (without much extra costs) where we consider as domain of our functions $f$ certain metric spaces $X$ (and in some cases we will consider such metric spaces $Y$ for the images as well). In this general case the intuitive notion of plottability is no longer meaningful. However, our formal notion of recursiveness is still available and has important applications in theory. Altogether, we arrive at the following problem:

Non-uniform Graph Problem. Which computable metric spaces $X$ and $Y$ have the property that for all continuous functions $f: X \rightarrow Y$ it holds that $f$ is computable, if and only if $\operatorname{graph}(f)=\{(x, f(x)): x \in X\} \subseteq X \times Y$ is recursive?

This non-uniform question relates a computability property of $f$ just logically with a computability property of graph $(f)$. But given $f$, can we effectively compute graph $(f)$ from $f$ ? And, vice versa, given graph $(f)$, can we effectively compute $f$ ? This uniform question 
can be formulated as follows.

Uniform Graph Problem. Which computable metric spaces $X$ and $Y$ have the property that the mapping graph $: \mathcal{C}(X, Y) \rightarrow \mathcal{A}(X \times Y), f \mapsto \operatorname{graph}(f)$ and its inverse are computable?

Here, by $\mathcal{C}(X, Y)$ we denote the set of continuous functions $f: X \rightarrow Y$ and by $\mathcal{A}(X \times Y)$ the set of closed subsets of $X \times Y$.

We are far from presenting a complete solution of the above questions and we will mainly concentrate on the case $Y=\mathbb{R}^{n}$. Even in this special case it appears that the answers to these questions sensitively rely on topological and computational properties of the space $X$ and even in case of important and well-understood concrete spaces $X$ we obtain a somewhat surprising variety of answers (see Figure 5).

We close this introduction with a short survey on the organization of this paper. In the following Section 2 we will introduce some basic concepts of computable analysis and Section 3 is devoted to computable metric spaces. To make all the above problems precise, we have, for instance, to define computability on the hyperspace $\mathcal{A}(X \times Y)$ of closed subsets and we have to define an appropriate notion of recursiveness. The corresponding definitions will be introduced in Section 4. In Section 5 we discuss one direction of the Graph Theorem which we will call weak Graph Theorem: any total computable function $f: X \rightarrow Y$ has a recursive graph and this holds even uniformly. In Section 6, 7, 8 and 9 we discuss different properties on computable metric spaces which guarantee that the inverse direction of the Graph Theorem holds as well. Such conditions are recursive compactness of the target space or different effective connectedness properties of the source space. Finally, in the Conclusions we give a survey of our results.

\section{Preliminaries from computable analysis}

In this section we briefly summarize some notions from computable analysis. For details the reader is refered to [18]. The basic idea of the representation based approach to computable analysis is to represent infinite objects like real numbers, functions or sets, by infinite strings over some alphabet $\Sigma$ (which should at least contain the symbols 0 and 1). Thus, a representation of a set $X$ is a surjective mapping $\delta: \subseteq \Sigma^{\omega} \rightarrow X$ and in this situation we will call $(X, \delta)$ a represented space. Here $\Sigma^{\omega}$ denotes the set of infinite sequences over $\Sigma$ and the inclusion symbol is used to indicate that the mapping might be partial. If we have two represented spaces, then we can define the notion of a computable function.

Definition 2.1 (Computable function) Let $(X, \delta)$ and $\left(Y, \delta^{\prime}\right)$ be represented spaces. A function $f: \subseteq X \rightarrow Y$ is called $\left(\delta, \delta^{\prime}\right)$-computable, if there exists some computable function $F: \subseteq \Sigma^{\omega} \rightarrow \Sigma^{\omega}$ such that $\delta^{\prime} F(p)=f \delta(p)$ for all $p \in \operatorname{dom}(f \delta)$. 
Of course, we have to define computability of functions $F: \subseteq \Sigma^{\omega} \rightarrow \Sigma^{\omega}$ to make this definition complete, but this can be done via Turing machines: $F$ is computable if there exists some Turing machine, which computes infinitely long and transforms each sequence $p$, written on the input tape, into the corresponding sequence $F(p)$, written on the one-way output tape. Later on, we will also need computable multi-valued operations $f: \subseteq X \rightrightarrows Y$, which are defined analogously to computable functions by substituting $\delta^{\prime} F(p) \in f \delta(p)$ for the equation in Definition 2.1 above. If the represented spaces are fixed or clear from the context, then we will simply call a function or operation $f$ computable.

Analogously to the notion of computability we can define the notion of $\left(\delta, \delta^{\prime}\right)$-continuity for single- and multi-valued operations, by substituting a continuous function $F: \subseteq \Sigma^{\omega} \rightarrow \Sigma^{\omega}$ for the computable function $F$ in the definitions above. On $\Sigma^{\omega}$ we use the Cantor topology, which is simply the product topology of the discrete topology on $\Sigma$. Again we will simply say that the corresponding function is continuous, if the representations are fixed or clear from the context. If not mentioned otherwise, we will always assume that a represented space is endowed with the final topology induced by its representation.

This will lead to no confusion with the ordinary topological notion of continuity, as long as we are dealing with admissible representations. A representation $\delta$ of a topological space $X$ is called admissible, if $\delta$ is maximal among all continuous representations $\delta^{\prime}$ of $X$ in a certain sense. If $\delta, \delta^{\prime}$ are admissible representations of $T_{0}$-spaces with countable bases, $X$, $Y$, then a function $f: \subseteq X \rightarrow Y$ is $\left(\delta, \delta^{\prime}\right)$-continuous, if and only if it is continuous in the ordinary topological sense.

Given a represented space $(X, \delta)$, we will occasionally use the notions of a computable sequence and a computable point. A computable sequence is a computable function $f: \mathbb{N} \rightarrow X$, where we assume that $\mathbb{N}=\{0,1,2, \ldots\}$ is represented by $\delta_{\mathbb{N}}\left(1^{n} 0^{\omega}\right):=n$ and a point $x \in X$ is called computable, if there is a constant computable function with value $x$.

Given two represented spaces $(X, \delta)$ and $\left(Y, \delta^{\prime}\right)$, there is a canonical representation $\left[\delta, \delta^{\prime}\right]$ of $X \times Y$ and a representation $\left[\delta \rightarrow \delta^{\prime}\right]$ of certain functions $f: X \rightarrow Y$. If $\delta, \delta^{\prime}$ are admissible representations of $T_{0}$-spaces with countable bases, then $\left[\delta \rightarrow \delta^{\prime}\right]$ is actually a representation of the set $\mathcal{C}(X, Y)$ of continuous functions $f: X \rightarrow Y$. If $Y=\mathbb{R}$, then we write for short $\mathcal{C}(X):=\mathcal{C}(X, \mathbb{R})$. The function space representation can be characterized by the fact that it admits evaluation and type conversion.

Proposition 2.2 (Evaluation and type conversion) Let $(X, \delta),\left(Y, \delta^{\prime}\right)$ be admissibly represented $T_{0}$-spaces with countable bases and let $\left(Z, \delta^{\prime \prime}\right)$ be a represented space. Then:

(1) (Evaluation) ev : $\mathcal{C}(X, Y) \times X \rightarrow Y,(f, x) \mapsto f(x)$ is $\left(\left[\left[\delta \rightarrow \delta^{\prime}\right], \delta\right], \delta^{\prime}\right)$-computable,

(2) (Type conversion) $f: Z \times X \rightarrow Y$, is $\left(\left[\delta^{\prime \prime}, \delta\right], \delta^{\prime}\right)$-computable, if and only if the function $\check{f}: Z \rightarrow \mathcal{C}(X, Y)$, defined by $\check{f}(z)(x):=f(z, x)$ is $\left(\delta^{\prime \prime},\left[\delta \rightarrow \delta^{\prime}\right]\right)$-computable.

The proof of this proposition is based on a version of smn- and utm-Theorem and can be found in [18]. If $(X, \delta),\left(Y, \delta^{\prime}\right)$ are admissibly represented $T_{0}$-spaces with countable bases, 
then in the following we will always assume that $\mathcal{C}(X, Y)$ is represented by $\left[\delta \rightarrow \delta^{\prime}\right]$. It is known that the computable points in $\left(\mathcal{C}(X, Y),\left[\delta \rightarrow \delta^{\prime}\right]\right)$ are just the $\left(\delta, \delta^{\prime}\right)$-computable functions $f: X \rightarrow Y$ [18]. If $(X, \delta)$ is a represented space, then we will always assume that the set of sequences $X^{\mathbb{N}}$ is represented by $\delta^{\mathbb{N}}:=\left[\delta_{\mathbb{N}} \rightarrow \delta\right]$. The computable points in $\left(X^{\mathbb{N}}, \delta^{\mathbb{N}}\right)$ are just the computable sequences in $(X, \delta)$. Moreover, we assume that $X^{n}$ is always represented by $\delta^{n}$, which can be defined inductively by $\delta^{1}:=\delta$ and $\delta^{n+1}:=\left[\delta^{n}, \delta\right]$.

\section{Computable metric spaces}

In this section we will briefly discuss computable metric spaces, see also [4] for further details. First, we just mention that we will denote the open balls of a metric space $(X, d)$ by $B(x, \varepsilon):=\{y \in X: d(x, y)<\varepsilon\}$ for all $x \in X, \varepsilon>0$ and correspondingly the closed balls by $\bar{B}(x, \varepsilon):=\{y \in X: d(x, y) \leq \varepsilon\}$. Occasionally, we denote complements of sets $A \subseteq X$ by $A^{c}:=X \backslash A$.

Definition 3.1 (Computable metric space) A tuple $(X, d, \alpha)$ is called a computable metric space, if

(1) $d: X \times X \rightarrow \mathbb{R}$ is a metric on $X$,

(2) $\alpha: \mathbb{N} \rightarrow X$ is a sequence which is dense in $X$,

(3) $d \circ(\alpha \times \alpha): \mathbb{N}^{2} \rightarrow \mathbb{R}$ is a computable (double) sequence in $\mathbb{R}$.

Here, we tacitly assume that the reader is familiar with the notion of a computable sequence of reals, but we will come back to that point below. Occasionally, we will say for short that $X$ is a computable metric space. Obviously, a computable metric space is especially separable. Given a computable metric space $(X, d, \alpha)$, its Cauchy representation $\delta_{X}: \subseteq \Sigma^{\omega} \rightarrow X$ can be defined by

$$
\delta_{X}\left(01^{n_{0}+1} 01^{n_{1}+1} 01^{n_{2}+1} \ldots\right):=\lim _{i \rightarrow \infty} \alpha\left(n_{i}\right)
$$

for all $n_{i}$ such that $\left(\alpha\left(n_{i}\right)\right)_{i \in \mathbb{N}}$ converges and $d\left(\alpha\left(n_{i}\right), \alpha\left(n_{j}\right)\right) \leq 2^{-i}$ for all $j>i$ (and undefined for all other input sequences). In the following we tacitly assume that computable metric spaces are represented by their Cauchy representations. If $X$ is a computable metric space, then it is easy to see that $d: X \times X \rightarrow \mathbb{R}$ is computable (see Proposition 5.3 in [4]). All Cauchy representations are admissible with respect to the corresponding metric topology.

An important computable metric space is $\left(\mathbb{R}, d_{\mathbb{R}}, \alpha_{\mathbb{R}}\right)$ with the Euclidean metric $d_{\mathbb{R}}(x, y):=$ $|x-y|$ and some numbering $\alpha_{\mathbb{R}}\langle i, j, k\rangle:=(i-j) /(k+1)$ of the rational numbers $\mathbb{Q}$. Here, $\langle i, j\rangle:=1 / 2(i+j)(i+j+1)+j$ denotes Cantor pairs and this definition is extended inductively to finite tuples. For short we will occasionally write $\bar{k}:=\alpha_{\mathbb{R}}(k)$. In the following we assume that $\mathbb{R}$ is endowed with the Cauchy representation $\delta_{\mathbb{R}}$ induced by the computable metric space given above. This representation of $\mathbb{R}$ can also be defined, if ( $\left.\mathbb{R}, d_{\mathbb{R}}, \alpha_{\mathbb{R}}\right)$ just fulfills (1) and (2) of the definition above and this leads to a definition of computable 
real number sequences without circularity. Occasionally, we will also use the represented $\operatorname{space}\left(\mathbb{Q}, \delta_{\mathbb{Q}}\right)$ of rational numbers with $\delta_{\mathbb{Q}}\left(1^{n} 0^{\omega}\right):=\alpha_{\mathbb{R}}(n)=\bar{n}$. We close this section with a brief discussion of metric product spaces.

Proposition 3.2 (Product spaces) If $(X, d, \alpha),\left(Y, d^{\prime}, \alpha^{\prime}\right)$ are computable metric spaces, then the product space $\left(X \times Y, d^{\prime \prime}, \alpha^{\prime \prime}\right)$, defined by

$$
d^{\prime \prime}\left((x, y),\left(x^{\prime}, y^{\prime}\right)\right):=\max \left\{d\left(x, x^{\prime}\right), d^{\prime}\left(y, y^{\prime}\right)\right\} \text { and } \alpha^{\prime \prime}\langle i, j\rangle:=\left(\alpha(i), \alpha^{\prime}(j)\right),
$$

is a computable metric space too and the canonical projections of the product space $\operatorname{pr}_{1}: X \times Y \rightarrow X$ and $\operatorname{pr}_{2}: X \times Y \rightarrow Y$ are computable.

Especially, the corresponding Cauchy representations $\delta_{X \times Y}$ and $\left[\delta_{X}, \delta_{Y}\right]$ are computably equivalent. In the following we will tacitly use the computable metric spaces $\mathbb{R}^{n},\{0,1\}^{\mathbb{N}}$ and $\mathbb{N}^{\mathbb{N}}$, defined in the standard way.

\section{Hyperspaces of closed subsets}

In this section we want to introduce computability on the hyperspace of closed subsets of computable metric spaces. At this point we have to consider carefully the fact that different equivalent characterizations of the notion of a recursive closed subset $A \subseteq \mathbb{R} \times \mathbb{R}$ split into inequivalent notions if we generalize them to computable metric spaces. The logical relation between several notions of effectivity of closed subsets of metric spaces is visualized in Figure 3.

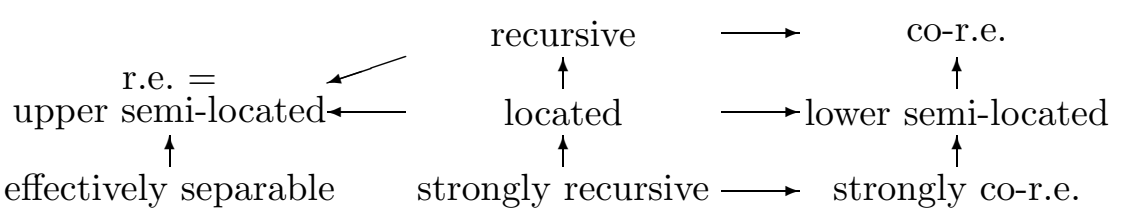

Fig. 3. Notions of effectivity for closed subsets of computable metric spaces.

The displayed results have been obtained in [7] from a very uniform point of view and each arrow in the diagram does not only indicate an implication but an effective reducibility. Below, we will precisely define the notions which are relevant to the present paper. In case of Euclidean space (and certain other computable metric spaces) the vertical arrows can be reversed and thus the three horizontal layers of the diagram collapse [8]. However, some examples prove that these notions have to be distinguished in the general case of computable metric spaces $[7,3]$. If we consider a subset $A \subseteq \mathbb{N}$ as a closed subset of $\mathbb{R}$ (embedded in the canonical way), then the set is r.e., co-r.e. and recursive in the classical sense, if and only if it is r.e., co-r.e. and recursive in the metric sense, respectively. Thus, all the displayed notions can be considered as formal generalizations of the corresponding classical notions of r.e., co-r.e. and recursive sets. 
Now the question appears, which of these notions fit together with the Graph Theorem. It could happen, for instance, that the graph of a computable function is recursive (but not strongly recursive in general), while only continuous functions with a strongly recursive graph are computable in general. However, our results show that this does not happen and actually the notion "recursive" is the one which optimally fits to the Graph Theorem. In the following definition we start to define corresponding hyperspace representations. Such representations have been studied in the Euclidean case in $[8,18]$ and for the metric case in [7].

Definition 4.1 (Hyperspace of closed subsets) Let $(X, d, \alpha)$ be a computable metric space. We endow the hyperspace $\mathcal{A}(X):=\{A \subseteq X: A$ closed $\}$ of closed subsets with the representation $\delta_{\mathcal{A}(X)}^{<}$, defined by

$$
\begin{gathered}
\delta_{\mathcal{A}(X)}^{<}\left(01^{\left\langle n_{0}, k_{0}\right\rangle+1} 01^{\left\langle n_{1}, k_{1}\right\rangle+1} 01^{\left\langle n_{2}, k_{2}\right\rangle+1} \ldots\right)=A \\
: \Longleftrightarrow\{(n, k): A \cap B(\alpha(n), \bar{k}) \neq \emptyset\}=\left\{\left(n_{i}, k_{i}\right) \in \mathbb{N}^{2}: i \in \mathbb{N}\right\},
\end{gathered}
$$

and with the representation $\delta_{\mathcal{A}(X)}^{>}$, defined by

$$
\delta_{\mathcal{A}(X)}^{>}\left(01^{\left\langle n_{0}, k_{0}\right\rangle+1} 01^{\left\langle n_{1}, k_{1}\right\rangle+1} 01^{\left\langle n_{2}, k_{2}\right\rangle+1} \ldots\right):=X \backslash \bigcup_{i=0}^{\infty} B\left(\alpha\left(n_{i}\right), \overline{k_{i}}\right) .
$$

Whenever we have two representations $\delta, \delta^{\prime}$ of some set, we can define the infimum $\delta \sqcap \delta^{\prime}$ of $\delta$ and $\delta^{\prime}$ by $\left(\delta \sqcap \delta^{\prime}\right)\langle p, q\rangle=x: \Longleftrightarrow \delta(p)=x$ and $\delta^{\prime}(q)=x$. We use the short notations $\mathcal{A}_{<}=\mathcal{A}_{<}(X)=\left(\mathcal{A}_{<}(X), \delta_{\mathcal{A}(X)}^{<}\right), \mathcal{A}_{>}=\mathcal{A}_{>}(X)=\left(\mathcal{A}_{>}(X), \delta_{\mathcal{A}(X)}^{>}\right)$and $\mathcal{A}=\mathcal{A}(X)=$ $\left(\mathcal{A}(X), \delta_{\mathcal{A}(X)}^{<} \sqcap \delta_{\mathcal{A}(X)}^{>}\right)$for the corresponding represented spaces. For the computable points of these spaces special names are used.

Definition 4.2 (Recursively enumerable and recursive sets) Let $X$ be a computable metric space and let $A \subseteq X$ be a closed subset.

(1) $A$ is called r.e. closed, if $A$ is a computable point in $\mathcal{A}_{<}(X)$,

(2) $A$ is called co-r.e. closed, if $A$ is a computable point in $\mathcal{A}_{>}(X)$,

(3) $A$ is called recursive closed, if $A$ is a computable point in $\mathcal{A}(X)$.

We continue this section with some helpful results on hyperspaces, which follow directly from results in [7]. The first result states that we can represent closed subsets by preimages of continuous functions. It is an effective version of the statement that closed subsets of metric spaces coincide with the functional closed subsets.

Proposition 4.3 (Functional closed subsets) Let $X$ be a computable metric space. The map $Z: \mathcal{C}(X, \mathbb{R}) \rightarrow \mathcal{A}_{>}(X), f \mapsto f^{-1}\{0\}$ is computable and admits a computable right-inverse $\mathcal{A}_{>}(X) \rightrightarrows \mathcal{C}(X, \mathbb{R})$.

The second result can be considered as an effective version of the statement that closed subsets of separable metric spaces are separable. Here and in the following $\bar{A}$ denotes the topological closure of a subset $A \subseteq X$ of some topological space $X$. 
Proposition 4.4 (Separable closed subsets) Let $X$ be a computable metric space. The mapping range $: X^{\mathbb{N}} \rightarrow \mathcal{A}_{<}(X),\left(x_{n}\right)_{n \in \mathbb{N}} \mapsto \overline{\left\{x_{n}: n \in \mathbb{N}\right\}}$ is computable and if $X$ is complete, then it admits a computable multi-valued partial right-inverse $\subseteq \mathcal{A}_{<}(X) \rightrightarrows X^{\mathbb{N}}$, defined for all non-empty closed subsets.

We close this section with the definition of the remaining notions of effectivity for closed subsets which we are going to use in this paper.

Definition 4.5 (Strongly recursive and located subsets) Let $(X, d, \alpha)$ be a computable metric space and let $A \subseteq X$ be a closed subset. Then

(1) $A$ is called strongly co-r.e. closed, if $\{\langle n, k\rangle \in \mathbb{N}: A \cap \bar{B}(\alpha(n), \bar{k})=\emptyset\}$ is r.e.

(2) $A$ is called strongly recursive closed, if $A$ is r.e. closed and strongly co-r.e. closed.

(3) $A$ is called located, if $A=\emptyset$ or the distance function $d_{A}: X \rightarrow \mathbb{R}, x \mapsto \inf _{y \in X} d(x, y)$ of $A$ is computable.

\section{The weak Graph Theorem}

In this section we will study the easy direction of the Graph Theorem and we will discuss a number of counterexamples which limit the possibilities for the problematic direction. We immediately obtain the following positive result, which is based on Propositions 4.4 and 4.3 .

Theorem 5.1 (Weak Graph Theorem) Let $X$ and $Y$ be computable metric spaces. The mapping graph $: \mathcal{C}(X, Y) \rightarrow \mathcal{A}(X \times Y), f \mapsto \operatorname{graph}(f)$ is computable.

Proof. We consider the computable metric spaces $(X, d, \alpha),\left(Y, d^{\prime}, \beta\right)$. Then, $\alpha: \mathbb{N} \rightarrow X$ is a computable function such that $\overline{\operatorname{range}(\alpha)}=X$. If $f: X \rightarrow Y$ is continuous, then $S: X \rightarrow X \times Y, x \mapsto(x, f(x))$ is continuous too and because of continuity of $S$ we obtain that range $(S \alpha)$ is dense in range $(S)=\operatorname{graph}(f)$. Using evaluation and type conversion as well as Proposition 4.4, it follows that graph : $\mathcal{C}(X, Y) \rightarrow \mathcal{A}_{<}(X \times Y)$ is computable.

Now $U: X \times Y \rightarrow \mathbb{R},(x, y) \mapsto d^{\prime}(f(x), y)$ is continuous, since $f: X \rightarrow Y$ and the metric $d^{\prime}: Y \times Y \rightarrow \mathbb{R}$ are continuous and we obtain

$$
U(x, y)=0 \Longleftrightarrow d^{\prime}(f(x), y)=0 \Longleftrightarrow f(x)=y \Longleftrightarrow(x, y) \in \operatorname{graph}(f) .
$$

Thus $U^{-1}\{0\}=\operatorname{graph}(f)$ and evaluation together with type conversion and Proposition 4.3 allow to conclude that graph $: \mathcal{C}(X, Y) \rightarrow \mathcal{A}_{>}(X \times Y)$ is computable. Altogether, this shows that graph $: \mathcal{C}(X, Y) \rightarrow \mathcal{A}(X \times Y)$ is computable.

Unfortunately, the partial inverse graph ${ }^{-1}$ is not continuous in general, as the following result shows. Here and in the following we write $w \sqsubseteq p$ if $w$ is a finite prefix of $p$. 
Proposition 5.2 The function graph $^{-1}: \subseteq \mathcal{A}(X \times \mathbb{R}) \rightarrow \mathcal{C}(X)$ is discontinuous in case of Cantor space $X=\{0,1\}^{\mathbb{N}}$.

Proof. Let $f_{n}:\{0,1\}^{\mathbb{N}} \rightarrow \mathbb{R}$ be defined by

$$
f_{n}(p):=\left\{\begin{array}{l}
n+1 \text { if } 0^{n} \sqsubseteq p \\
0 \quad \text { else }
\end{array}\right.
$$

for all $n \in \mathbb{N}$ and $p \in\{0,1\}^{\mathbb{N}}$. Then $\left(f_{n}\right)_{n \in \mathbb{N}}$ is a sequence in $\mathcal{C}\left(\{0,1\}^{\mathbb{N}}\right)$ which does not converge, but $\left(\operatorname{graph}\left(f_{n}\right)\right)_{n \in \mathbb{N}}$ converges in $\mathcal{A}\left(\{0,1\}^{\mathbb{N}} \times \mathbb{R}\right)$ to $A:=\{0,1\}^{\mathbb{N}} \times\{0\}$ (since any positive or negative information on $A$ is shared by almost all sets in the sequence).

Thus, the uniform version of the Graph Theorem does not hold true in general, not even for compact computable metric spaces $X$. However, as Corollary 7.3 will show, this fact cannot be used to construct a computable counterexample for the non-uniform case. Such a non-uniform counterexample can be obtained if we omit compactness as the following example shows.

Example 5.3 Let $X=\mathbb{N}^{\mathbb{N}}$ be the Baire space. There exists a continuous but noncomputable function $f: X \rightarrow \mathbb{R}$ such that graph $(f) \subseteq X \times \mathbb{R}$ is strongly recursive closed. The same holds in case of $X=\mathbb{N}\{0,1\}^{\mathbb{N}}$.

Proof. Let $a: \mathbb{N} \rightarrow \mathbb{N}$ be some computable and injective enumeration of a non-recursive set $K=\operatorname{range}(a)$. Define $f: \mathbb{N}^{\mathbb{N}} \rightarrow \mathbb{R}$ by

$$
f(n p):= \begin{cases}k+1 & \text { if there exists some } k \text { with } a(k)=n \text { and } 0^{k} \sqsubseteq p \\ 0 \quad & \text { otherwise }\end{cases}
$$

for all $p \in \mathbb{N}^{\mathbb{N}}$ and $n \in \mathbb{N}$. Then $f$ is continuous but non-computable since $K=\operatorname{range}(a)$ is not co-r.e. and

$$
n \notin K \Longleftrightarrow f\left(n 0^{\omega}\right)<1
$$

Moreover,

$$
\begin{gathered}
\left(w \mathbb{N}^{\mathbb{N}} \times(r, s)\right) \cap \operatorname{graph}(f) \neq \emptyset \\
\Longleftrightarrow(\exists n, k \geq 0, j \geq 1)\left(\left(\left(w \sqsubseteq n 0^{k} \text { or } n 0^{k} \sqsubseteq w\right) \text { and } a(k)=n \text { and } k+1 \in(r, s)\right)\right. \text { or } \\
\left.\left(\left(w=n 0^{k} \text { or } n 0^{k} j \sqsubseteq w\right) \text { and } n \notin\{a(0), \ldots, a(k)\} \text { and } 0 \in(r, s)\right)\right)
\end{gathered}
$$

for all $w \in \mathbb{N}^{*}$ and $r, s \in \mathbb{Q}$ with $r \leq s$. Since $a$ is computable and only finitely many values $n, k, j$ have to be checked, it follows that the stated property is recursive in $r, s$ 
and $w$. Especially, graph $(f)$ is r.e. closed. Analogously, the equivalence also holds with $[r, s]$ substituted for $(r, s)$ in all of its three incidences. Thus, graph $(f)$ is also strongly co-r.e. closed. Altogether, graph $(f)$ is strongly recursive closed. The proof for the case $X=\mathbb{N}\{0,1\}^{\mathbb{N}}$ is the same.

Altogether, this shows that the problematic direction of the Graph Theorem does not hold in general, neither in the uniform case nor in the non-uniform case. The previous example additionally shows that in general it does not help to use the stronger notion of "strongly recursive closed subsets" for the problematic direction (not even in the non-uniform case).

Finally we ask, whether the positive result of this section could be improved by obtaining a stronger computability property of the graph. On the one hand, results of Cenzer and Remmel show that there is a computable function $f: \mathbb{N}^{\mathbb{N}} \rightarrow \mathbb{N}^{\mathbb{N}}$ such that $\operatorname{graph}(f)$ is not strongly recursive (see Corollary 4.6 in [9]). On the other hand, a reinterpretation of Example 1.3 shows that the graph of a computable function is not located in general. For the reformulation we consider $X:=\mathbb{R} \backslash \mathbb{N}$ as a computable metric subspace of $\mathbb{R}$, endowed with the restriction of the Euclidean metric to $X \times X$.

Example 5.4 Consider $X=\mathbb{R} \backslash \mathbb{N}$ as a computable metric subspace of $\mathbb{R}$ endowed with the restriction of the Euclidean metric. There exists a computable function $f: X \rightarrow \mathbb{R}$ such that $\operatorname{graph}(f) \subseteq X \times \mathbb{R}$ is not located.

Since graph $(f)$ needs not to be closed as a subset of $\mathbb{R} \times \mathbb{R}$ we could even obtain the same result with $[0,1]$ as target space instead of $\mathbb{R}$ by a direct construction. The reader should notice the fine distinctions: graph $(f)$ is recursive as a closed subset of $X \times \mathbb{R}$ by Theorem 5.1 but it is not recursive as a closed subset of $\mathbb{R} \times \mathbb{R}$ by Example 1.3. Moreover, it is not located as subset of $X \times \mathbb{R}$ and thus, in particular, not strongly recursive in the same sense (however, there is a different but equivalent metric on $X$ which yields the forementioned properties). Therefore, Theorem 5.1 formulates the best possible general result (with respect to the computability properties of subsets given in Figure 3).

\section{Compact target spaces}

In this section we want to prove that the inverse graph ${ }^{-1}$ of the graph mapping becomes computable if the target space is effectively compact in a certain sense. We start with the definition of some effectiveness notions for compact subsets (see [7]).

Definition 6.1 (Recursive compact sets) Let $(X, d, \alpha)$ be a computable metric space. A subset $K \subseteq X$ is called co-r.e. compact, if it is compact and the set

$$
\left\{\left\langle\left\langle n_{0}, k_{0}\right\rangle,\left\langle n_{1}, k_{1}\right\rangle, \ldots,\left\langle n_{j}, k_{j}\right\rangle, j\right\rangle \in \mathbb{N}: K \subseteq \bigcup_{i=0}^{j} B\left(\alpha\left(n_{i}\right), \overline{k_{i}}\right)\right\}
$$


is r.e. Moreover, a subset $K \subseteq X$ is called recursive compact, if it is both, r.e. closed and co-r.e. compact.

One can prove that for computable metric spaces which fulfill certain additional properties, a compact subset $K \subseteq X$ is co-r.e. compact, if and only if it is co-r.e. closed [7]. We will call a computable metric space $Y$ recursive compact, if it is recursive compact as a subset of itself. Any computable metric space $Y$ which is co-r.e. compact as a subset of itself is automatically recursive compact, since any computable metric space is r.e. closed as a subset of itself.

Theorem 6.2 (Compact Graph Theorem) Let $X$ and $Y$ be computable metric spaces and let $Y$ be co-r.e. compact. Then the graph map graph : $\mathcal{C}(X, Y) \rightarrow \mathcal{A}_{>}(X \times Y)$, $f \mapsto \operatorname{graph}(f)$ as well as its partial inverse

$$
\operatorname{graph}^{-1}: \subseteq \mathcal{A}_{>}(X \times Y) \rightarrow \mathcal{C}(X, Y)
$$

are computable.

Proof. The first part of the claim follows from the weak Graph Theorem 5.1. Now any continuous function $f: X \rightarrow Y$ can be represented as

$$
f(x)=\operatorname{in}_{Y}^{-1} \circ \sec (\operatorname{graph}(f), x)
$$

for any $x \in X$, where sec $: \mathcal{A}_{>}(X \times Y) \times X \rightarrow \mathcal{A}_{>}(Y)$ and in $\operatorname{in}_{Y}: Y \rightarrow \mathcal{A}_{>}(Y)$ are the mappings defined in Lemma 6.3 and Lemma 6.4. Since sec and $\operatorname{in}_{Y}^{-1}$ are computable, it follows by type conversion that graph ${ }^{-1}$ is computable.

It remains to prove the following two lemmas.

Lemma 6.3 Let $X, Y$ be computable metric spaces. Then the section map

$$
\sec : \mathcal{A}_{>}(X \times Y) \times X \rightarrow \mathcal{A}_{>}(Y),(A, x) \mapsto A_{x}:=\{y \in Y:(x, y) \in A\}
$$

is computable.

Proof. Let $A \subseteq X \times Y$ be a closed subset and let $\left(x_{i}, y_{i}\right) \in X \times Y$ and $r_{i} \in \mathbb{Q}$ for all $i \in \mathbb{N}$ be points such that $\bigcup_{i=0}^{\infty} B\left(\left(x_{i}, y_{i}\right), r_{i}\right)=(X \times Y) \backslash A$. Then one directly obtains $\bigcup\left\{B\left(y_{i}, r_{i}\right): i \in \mathbb{N}\right.$ and $\left.x \in B\left(x_{i}, r_{i}\right)\right\}=Y \backslash A_{x}$. Since the property " $x \in B\left(x_{i}, r_{i}\right)$ " is r.e., the claim follows.

The following lemma will be formulated slightly more general as necessary for the proof of Theorem 6.2 (the case $Z \neq Y$ will be used in order to prove Proposition 7.2 in the next section). 
Lemma 6.4 Let $Y$ be a computable metric space and let $Z \subseteq Y$ be recursive compact. The injection map $\operatorname{in}_{Z}: Z \rightarrow \mathcal{A}_{>}(Y), y \mapsto\{y\}$ and its partial inverse $\operatorname{in}_{Z}^{-1}: \subseteq \mathcal{A}_{>}(Y) \rightarrow Z$ are computable.

Proof. Consider the computable metric space $(Y, d, \alpha)$. Since $Z$ is r.e. closed we can consider it as computable metric subspace $\left(Z,\left.d\right|_{Z \times Z}, \beta\right)$ of $Y$. Without loss of generality we can assume range $(\beta) \subseteq$ range $(\alpha)$. Computability of the injection map $\operatorname{in}_{Z}$ follows directly since

$$
Y \backslash\{y\}=\bigcup\{B(\alpha(n), \bar{k}): n, k \in \mathbb{N} \text { and } d(y, \alpha(n))>\bar{k}\}
$$

for all $y \in Y$. Now let $y \in Z$ and assume that $x_{i} \in \operatorname{range}(\alpha)$ and $r_{i} \in \mathbb{Q}$ for all $i \in \mathbb{N}$ are points such that $Y \backslash\{y\}=\bigcup_{i=0}^{\infty} B\left(x_{i}, r_{i}\right)$. Then

$$
\begin{aligned}
y \in B(x, r) & \Longleftrightarrow Z \subseteq B(x, r) \cup \bigcup_{i=0}^{\infty} B\left(x_{i}, r_{i}\right) \\
& \Longleftrightarrow(\exists j) Z \subseteq B(x, r) \cup \bigcup_{i=0}^{j} B\left(x_{i}, r_{i}\right),
\end{aligned}
$$

for all $x \in \operatorname{range}(\beta)$ and $r \in \mathbb{Q}$ with $r>0$, where the last equivalence holds since $Z$ is compact. Since $Z$ is even co-r.e. compact, it follows that $\mathrm{in}_{Z}^{-1}$ is computable.

As a corollary of Theorem 5.1 and of Theorem 6.2 we immediately obtain the following non-uniform version of the Graph Theorem.

Corollary 6.5 Let $X, Y$ be computable metric space, let $Y$ be co-r.e. compact and let $f: X \rightarrow Y$ be a (continuous) function. Then $f$ is computable, if and only if $\operatorname{graph}(f)$ is co-r.e. closed, if and only if $\operatorname{graph}(f)$ is recursive closed.

The formula in the proof of Theorem 6.2 additionally shows that $f$ is automatically continuous, whenever graph $(f)$ is closed (under the assumptions of the previous corollary). Hence, the corollary remains true without the word "continuous". The previous corollary has been proved directly in case of $X=Y=[0,1]$ and $X=Y=\{0,1\}^{\mathbb{N}}$ by Cenzer and Remmel (see Theorem 4.7 in [9]). In these cases one can even replay co-r.e. by strongly co-r.e. Proposition 5.2 and Example 5.3 show that the compactness conditions are not superfluous in the previous results. The following example shows that co-r.e. closed graphs cannot be replaced by r.e. closed graphs in the previous corollary.

Example 6.6 Let $X=Y=[0,1]$. There exists a continuous but non-computable function $f: X \rightarrow Y$ such that graph $(f) \subseteq X \times Y$ is r.e. closed. The same holds in case of $X=Y=\mathbb{R}$.

Proof. Let $a: \mathbb{N} \rightarrow \mathbb{N}$ be some computable and injective enumeration of a non-recursive set $K=\operatorname{range}(a)$. We define a function $f:[0,1] \rightarrow[0,1]$ as follows. For any $n \in K=$ range $(a)$ let $p_{n}:\left[2^{-n-1}-2^{-n-3}, 2^{-n-1}+2^{-n-3}\right] \rightarrow \mathbb{R}$ be the rational polygon with vertices 


$$
\begin{aligned}
\left(2^{-n-1}-2^{-n-3}, 0\right), & \left(2^{-n-1}-2^{-n-3-a^{-1}(n)}, 0\right),\left(2^{-n-1}, 2^{-n}\right), \\
& \left(2^{-n-1}+2^{-n-3-a^{-1}(n)}, 0\right),\left(2^{-n-1}+2^{-n-3}, 0\right)
\end{aligned}
$$

and let

$$
f(x):= \begin{cases}p_{n}(x) & \text { if there exists some } n \in \operatorname{range}(a)=K \text { with } \\ & x \in\left[2^{-n-1}-2^{-n-3}, 2^{-n-1}+2^{-n-3}\right] \\ 0 \quad & \text { else }\end{cases}
$$

for all $x \in[0,1]$.

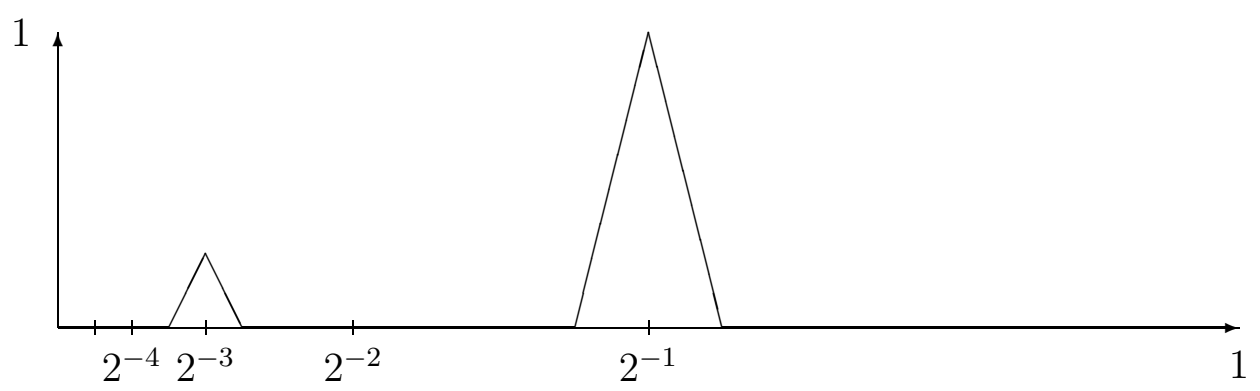

Fig. 4. The function $f$ in case of $a(0)=2, a(1)=0$ and $1,3,4,5,6, \ldots \notin K$.

It is easy to see that $f$ is continuous and $\operatorname{graph}(f) \subseteq[0,1] \times[0,1]$ is r.e. closed since $a$ is computable. On the other hand, $f$ is not computable, since $K=\operatorname{range}(a)$ is not co-r.e. and

$$
n \notin K \Longleftrightarrow f\left(2^{-n-1}\right)<2^{-n}
$$

Similar counterexamples have been presented by Cenzer and Remmel (also for the case $X=Y=\mathbb{N}^{\mathbb{N}}$, see Theorem 4.8 in [9]). As a further corollary of Theorem 5.1 and Theorem 6.2 we can formulate a uniform version of the statement that negative information on the graph of a continuous function with compact target can be translated into positive information.

Corollary 6.7 Let $X, Y$ be computable metric spaces and let $Y$ be co-r.e. compact. Then the identity id $: \mathcal{A}_{>}(X \times Y) \rightarrow \mathcal{A}(X \times Y), A \rightarrow A$, restricted to such subsets $A \subseteq X \times Y$ which are graphs of (continuous) functions $f: X \rightarrow Y$, is computable.

The following proposition shows that this does not hold in the general case of arbitrary computable metric spaces $Y$. 
Proposition 6.8 The function graph $^{-1}: \subseteq \mathcal{A}_{>}(X \times Y) \rightarrow \mathcal{C}(X, Y)$ is discontinuous in case of the unit interval $X=[0,1]$ and $Y=\mathbb{R}$. The same holds true with $\mathcal{A}_{<}$instead of $\mathcal{A}_{>}$(and in this case one can even choose $\left.Y=[0,1]\right)$.

Proof. Now let $f_{n}:[0,1] \rightarrow \mathbb{R}$ be defined by $f_{n}(x):=n$ for all $x \in[0,1]$ and $n \in \mathbb{N}$. Then $\left(f_{n}\right)_{n \in \mathbb{N}}$ is a sequence in $\mathcal{C}[0,1]$ which does not converge, but (graph $\left.\left(f_{n}\right)\right)_{n \in \mathbb{N}}$ converges in $\mathcal{A}_{>}([0,1] \times \mathbb{R})$ to $A:=[0,1] \times\{0\}$ (since any negative information on $A$ is shared by almost all sets in the sequence). This proves the claim for $\mathcal{A}_{>}$.

Let $f_{n}:[0,1] \rightarrow \mathbb{R}$ be defined by

$$
\begin{aligned}
\operatorname{graph}\left(f_{n}\right):= & \left\{\left(x, 2^{n+1} x\right): x \in\left[0,2^{-n-1}\right]\right\} \cup \\
& \left\{\left(x, 2-2^{n+1} x\right): x \in\left[2^{-n-1}, 2^{-n}\right]\right\} \cup\left(\left[2^{-n}, 1\right] \times\{0\}\right) .
\end{aligned}
$$

Then $\left(f_{n}\right)_{n \in \mathbb{N}}$ is a sequence of continuous functions in $\mathcal{C}[0,1]$ which does not converge, but the sequence $\left(\operatorname{graph}\left(f_{n}\right)\right)_{n \in \mathbb{N}}$ converges in $\mathcal{A}_{<}([0,1] \times \mathbb{R})$ to $A:=[0,1] \times\{0\}$ (since any positive information on $A$ is shared by almost all sets in the sequence). This proves the claim for $\mathcal{A}_{<}$. Since range $\left(f_{n}\right) \subseteq[0,1]$ the same holds true in case of $Y=[0,1]$.

The second part of the previous proposition shows that the previous corollary does not hold true, if we replace $\mathcal{A}_{>}$by $\mathcal{A}_{<}$, even not if both, $X$ and $Y$, are recursive compact.

\section{$7 \quad$ Locally compact target spaces}

In this section we will show that the non-uniform version of the compact Graph Theorem, i.e. Corollary 6.5, can be transfered to the case that the source space $X$ is compact and the target space $Y$ is effectively locally compact. Up to now different notions of "effective local compactness" have been used (see for instance [20,7]) and the terminology is not stable yet. In the next definition we do not want to propose a new version, but be just formulate a very weak property which should be met by most of the reasonable definitions and which is sufficient for our proofs.

Definition 7.1 (Effective local compactness) A computable metric space $X$ is called effectively locally compact, if for any compact subset $A \subseteq X$, there exists a recursive compact subset $K \subseteq X$ such that $A \subseteq K$.

It is obvious that for instance the Euclidean space $\mathbb{R}^{n}$ is effectively locally compact in this sense (since any closed ball is recursive compact). Next we will show that if the target space is effectively locally compact, then we can deduce that any continuous function with co-r.e. graph maps computable points to computable points. And furthermore, it is computable restricted to any r.e. compact subset. If $f: X \rightarrow Y$ is a total function and $A \subseteq X$, then we denote by $\left.f\right|_{A}: \subseteq X \rightarrow Y$ the restriction of $f$ to $A$, defined by 
$\left.f\right|_{A}(x):=f(x)$ and $\operatorname{dom}(f):=A$.

Proposition 7.2 Let $X, Y$ be computable metric spaces, let $Y$ be effectively locally compact and let $f: X \rightarrow Y$ be a continuous function. If $\operatorname{graph}(f)$ is co-r.e. closed, then $\left.f\right|_{K}$ is computable for any compact subset $K \subseteq X$. Especially, $f$ maps computable points to computable points.

Proof. Since $f$ is continuous, it follows that $f(K)$ is compact for any compact set $K \subseteq X$. Since $Y$ is effectively locally compact, there is a recursive compact set $L \subseteq Y$ such that $f(K) \subseteq L$. Especially, $L$ can be considered as a computable metric subspace of $Y$ and thus, the injection $L \hookrightarrow Y$ is computable. Now $f$ can be represented as

$$
f(x)=\operatorname{in}_{L}^{-1} \circ \sec (\operatorname{graph}(f), x)
$$

for any $x \in K$, where sec $: \mathcal{A}_{>}(X \times Y) \times X \rightarrow \mathcal{A}_{>}(Y)$ and $\operatorname{in}_{L}: L \rightarrow \mathcal{A}_{>}(Y)$ are the functions defined in Lemma 6.3 and Lemma 6.4. Since sec, in $_{L}^{-1}$ and $L \hookrightarrow Y$ are computable, the partial function $\left.f\right|_{K}: \subseteq X \rightarrow Y$ is computable. The last part of the claim holds since $K:=\{x\}$ is a compact subset for any $x \in X$.

We obtain the following corollary of the previous result and the weak Graph Theorem 5.1 for compact source spaces and effectively locally compact target spaces.

Corollary 7.3 Let $X, Y$ be computable metric spaces, let $X$ be compact, let $Y$ be effectively locally compact and let $f: X \rightarrow Y$ be a continuous function. Then $f$ is computable, if and only if graph $(f)$ is co-r.e. closed, if and only if $\operatorname{graph}(f)$ is recursive closed.

This corollary especially applies to continuous functions $f:[0,1] \rightarrow \mathbb{R}$. Proposition 6.8 shows that the uniform version of the first equivalence of the previous corollary does not hold true in general. Here, continuity of $f$ does not automatically follow if $\operatorname{graph}(f)$ is closed (see Example 4.3 in [9]). Finally, Example 5.3 shows that the previous corollary does not hold true for locally compact source spaces $X$. The next example proves that effective local compactness of the target space is not a superfluous property in the previous corollary. The example is a modified version of Example 6.6 and as target space we will use the computable metric space (see e.g. [6] for details):

$$
\ell_{2}:=\left\{x \in \mathbb{R}^{\mathbb{N}}:\|x\|_{2}<\infty\right\} \text { with }\left\|\left(x_{k}\right)_{k \in \mathbb{N}}\right\|_{2}:=\sqrt{\sum_{k=0}^{\infty}\left|x_{k}\right|^{2}} .
$$

Example 7.4 Let $X=[0,1]$ and $Y=\ell_{2}$. There exists a continuous but non-computable function $f: X \rightarrow Y$ such that $\operatorname{graph}(f) \subseteq X \times Y$ is recursive closed.

Proof. Let $a: \mathbb{N} \rightarrow \mathbb{N}$ be some computable and injective enumeration of a non-recursive set $K=\operatorname{range}(a)$. We define a function $f:[0,1] \rightarrow \ell_{2}$ using the polygons $p_{n}$ as defined in 
Example 6.6, as follows:

$$
f(x)(k):=\left\{\begin{array}{l}
p_{n}(x) \text { if } a(k)=n \text { and } x \in\left[2^{-n-1}-2^{-n-3}, 2^{-n-1}+2^{-n-3}\right]=: I_{n} \\
0 \quad \text { else }
\end{array}\right.
$$

for all $x \in[0,1]$ and $k \in \mathbb{N}$.

It is easy to see that $f$ is continuous and $\operatorname{graph}(f) \subseteq[0,1] \times \ell_{2}$ is r.e. closed since $a$ is computable. One can also show that graph $(f)$ is co-r.e. In case that $x \in I_{n}$ and we already know that $n \notin a(\{0, \ldots, k\})$, then we obtain $B((x, y), r) \subseteq \operatorname{graph}(f)^{c}$ for any $y=\left(y_{0}, y_{1}, \ldots, y_{k}, 0,0, \ldots\right) \in \ell_{2}$ with $\|y\|>0$ and $0<r<\min \left\{\|y\|, 2^{-n-4}\right\}$. Exploiting this idea, one can exhaust graph $(f)^{c}$ effectively by open balls and thus graph $(f)$ is co-r.e.

On the other hand, $f$ is not computable, since $K=\operatorname{range}(a)$ is not co-r.e. and

$$
n \notin K \Longleftrightarrow\left\|f\left(2^{-n-1}\right)\right\|_{2}<2^{-n}
$$

In the previous example $f:[0,1] \rightarrow \ell_{2}$ is continuous and thus $K:=f[0,1]$ is compact. However, $K$ cannot be co-r.e. compact by Corollary 6.5 and by Corollary $7.3 \ell_{2}$ is not effectively locally compact (in the weak sense of Definition 7.1). In particular, there is no recursive compact set $K^{\prime} \subseteq \ell_{2}$ such that $K \subseteq K^{\prime}$. This observation especially proves that pure compactness of $Y$ is not sufficient in Theorem 6.2 (and correspondingly for Corollary 6.5). Moreover, effective local compactness of $Y$ cannot be replaced by compactness in Corollary 7.3.

\section{Locally connected source spaces}

In this section we will discuss another property of the source space which guarantees that the uniform inverse direction of the Graph Theorem becomes computable. We will call a computable metric space $X$ effectively locally connected (cf. [14]) if for any point $x \in X$ and any open ball $B(x, r)$ we can effectively find some connected neighbourhood $U$ of $x$ which is contained in $B(x, r)$. For the precise formulation we employ the hyperspace $\mathcal{O}(X)$ of open subsets of $X$, represented by $\delta_{\mathcal{O}(X)}(p)=X \backslash \delta_{\mathcal{A}_{>}(X)}(p)$.

Definition 8.1 (Effective local connectedness) A computable metric space $X$ is called effectively locally connected, if there exists a computable multi-valued function $C: \subseteq X \times \mathbb{R} \rightrightarrows \mathcal{O}(X)$ such that for any $x \in X$ and $r>0$ there exists some $U \in C(x, r)$ and any such $U$ is connected and fulfills $x \in U \subseteq B(x, r)$.

One should mention that it would not suffice to consider balls $U=B(x, s)$ with some $s \leq r$ because it might happen that any such ball is not connected although there exists some connected (more complicated) $U$ (consider an appropriate spiral line in $\mathbb{R}^{2}$ such that any 
box with the same center as the spiral cuts the spiral into several distinct connectedness components).

The main result of this section will be based on the following lemma which employs the hyperspace $\mathcal{K}(X):=\{K \subseteq X: K$ non-empty and compact $\}$ of non-empty compact subsets of a metric space $X$, endowed with the Hausdorff metric $d_{\mathcal{K}}: \mathcal{K}(X) \times \mathcal{K}(X) \rightarrow \mathbb{R}$, defined by

$$
d_{\mathcal{K}}(A, B):=\max \left\{\sup _{a \in A} d_{B}(a), \sup _{b \in B} d_{A}(b)\right\}
$$

If $(X, d, \alpha)$ is a computable metric space, then a standard numbering $\alpha_{\mathcal{K}}$ of the set $\mathcal{Q}$ of finite subsets of range $(\alpha)$ can be defined by $\alpha_{\mathcal{K}}\left\langle k,\left\langle n_{0}, \ldots, n_{k}\right\rangle\right\rangle:=\left\{\alpha\left(n_{0}\right), \ldots, \alpha\left(n_{k}\right)\right\}$. It is easy to see that under these assumptions $\left(\mathcal{K}(X), d_{\mathcal{K}}, \alpha_{\mathcal{K}}\right)$ is a computable metric space too. In the following we assume that $\mathcal{K}(X)$ is endowed with the corresponding Cauchy representation $\delta_{\mathcal{K}}$. It is easy to prove that the canonical injection $\mathcal{K}(X) \hookrightarrow \mathcal{A}(X)$ is computable [7]. As a preparation of our main result we formulate a lemma. Here, $\partial A$ denotes the border of a subset $A \subseteq X$ of a metric space $X$.

Lemma 8.2 Let $n \geq 1$ a natural number. The function

$$
I: \subseteq \mathbb{R}^{n} \times \mathbb{R} \rightarrow \mathcal{K}\left(\mathbb{R}^{n}\right),(y, \varepsilon) \mapsto \partial B(y, \varepsilon),
$$

defined for all $(y, \varepsilon) \in \mathbb{R}^{n} \times \mathbb{R}$ with $\varepsilon>0$, is computable.

The proof is straightforward. Now we are prepared to prove the following theorem.

Theorem 8.3 (Locally connected Graph Theorem) Let $X$ be an effectively locally connected computable metric space and let $n \geq 1$ be a natural number. The graph mapping graph : $\mathcal{C}\left(X, \mathbb{R}^{n}\right) \rightarrow \mathcal{A}\left(X \times \mathbb{R}^{n}\right), f \mapsto \operatorname{graph}(f)$ as well as its partial inverse

$$
\operatorname{graph}^{-1}: \subseteq \mathcal{A}\left(X \times \mathbb{R}^{n}\right) \rightarrow \mathcal{C}\left(X, \mathbb{R}^{n}\right)
$$

are computable.

Proof. We consider the computable metric space $(X, d, \alpha)$. Computability of graph follows from Theorem 5.1. We investigate the mapping graph ${ }^{-1}$. Thus, let $f: X \rightarrow \mathbb{R}^{n}$ be a continuous function. We can assume that $\operatorname{graph}(f) \in \mathcal{A}\left(X \times \mathbb{R}^{n}\right)$ is given. Furthermore, let $x \in X$ and a precision $k \in \mathbb{N}$ be given. We prove that we can effectively evaluate $f(x)$ up to precision $2^{-k}$ which implies by type conversion the desired result. Since graph $(f)$ is given as point of $\mathcal{A}\left(X \times \mathbb{R}^{n}\right)$, we can, on the one hand, find points $\left(x_{i}, y_{i}\right) \in \operatorname{range}(\alpha) \times \mathbb{Q}^{n}$ and rational numbers $r_{i}>0$ such that

$$
\operatorname{graph}(f)^{c}=\left(X \times \mathbb{R}^{n}\right) \backslash \operatorname{graph}(f)=\bigcup_{i=0}^{\infty} B\left(\left(x_{i}, y_{i}\right), r_{i}\right)=\bigcup_{i=0}^{\infty}\left(B\left(x_{i}, r_{i}\right) \times B\left(y_{i}, r_{i}\right)\right)
$$


and, on the other hand, given some open set $U \in \mathcal{O}\left(X \times \mathbb{R}^{n}\right)$ we can effectively realize that $U \cap \operatorname{graph}(f) \neq \emptyset$, if this is the case. Given a compact subset $K \in \mathcal{K}\left(\mathbb{R}^{n}\right)$ such that $K \subseteq \bigcup_{i=0}^{\infty} B\left(y_{i}, r_{i}\right)$, the computable Heine-Borel Theorem (cf. Theorems 4.6 and 4.10 in [8]) ensures that we can effectively find a natural number $m \in \mathbb{N}$ such that $K \subseteq \bigcup_{i=0}^{m} B\left(y_{i}, r_{i}\right)$. Now the evaluation of $f(x)$ up to precision $2^{-k}$ works as follows: we systematically search for some $y \in \mathbb{Q}^{n}$, some rational numbers $r>0, t \geq 1$ and natural numbers $n_{0}, \ldots, n_{m} \in \mathbb{N}$ and we determine some $W \in C(x, r)$ such that

(1) $x \in W \subseteq B(x, r)$ and $W$ is connected and open,

(2) $U \cap \operatorname{graph}(f) \neq \emptyset$ with $U:=W \times B(y, r)$,

(3) $d\left(x_{n_{i}}, x\right)+r<r_{n_{i}}$ for all $i=0, \ldots, m$,

(4) $\partial B(y, t r) \subseteq \bigcup_{i=0}^{m} B\left(y_{n_{i}}, r_{n_{i}}\right)$,

(5) $\operatorname{tr}<2^{-k}$.

If for certain values $y, r, t, n_{0}, \ldots, n_{m}$ and $W \in C(x, r)$ the conditions (2) to (5) are fulfilled, then we can eventually realize this by the previous considerations, the previous lemma and since $X$ is effectively locally connected. Now we claim that such values always exist and $\|f(x)-y\|<2^{-k}$ if (1) to (5) are fulfilled.

Thus, let us first assume that (1) to (5) hold. It is obvious that (3) implies $B(x, r) \subseteq$ $\bigcap_{i=0}^{m} B\left(x_{n_{i}}, r_{n_{i}}\right)$ and thus by (4) it follows

$$
B(x, r) \times \partial B(y, t r) \subseteq \bigcup_{i=0}^{m}\left(B\left(x_{n_{i}}, r_{n_{i}}\right) \times B\left(y_{n_{i}}, r_{n_{i}}\right)\right) \subseteq \operatorname{graph}(f)^{c}
$$

Thus, by (1) we obtain $f(W) \subseteq B(y, t r) \cup\left(\mathbb{R}^{n} \backslash \bar{B}(y, t r)\right)$ and hence $f(W)$ is covered by a disjoint union of open subsets. Since $W$ is connected and $f$ is continuous, it follows that $f(W)$ is connected too and thus $f(W)$ has empty intersection with either $B(y, t r)$ or $\mathbb{R}^{n} \backslash \bar{B}(y, t r)$. By (2) there is some $z \in W$ such that $f(z) \in B(y, r) \subseteq B(y, t r)$ and hence $f(W) \cap \bar{B}(y, t r)^{c}=\emptyset$. This implies $f(x) \in B(y, t r)$ by (1) and hence $\|f(x)-y\|<t r<2^{-k}$ by (5).

Now we still have to prove that there are always values $y, r, t, n_{0}, \ldots, n_{m}$ such that (2) to (5) are fulfilled with $W \in C(x, r)$. Therefore, let $0<s<2^{-k}$ be a rational number. Then $\partial B(f(x), s) \subseteq \bigcup\left\{B\left(y_{i}, r_{i}\right): i \in \mathbb{N}\right.$ and $\left.x \in B\left(x_{i}, r_{i}\right)\right\}$ and by compactness there exist $n_{0}, \ldots, n_{m} \in \mathbb{N}$ such that $\partial B(f(x), s) \subseteq \bigcup_{i=0}^{m} B\left(y_{n_{i}}, r_{n_{i}}\right)$ and $x \in \bigcap_{i=0}^{m} B\left(x_{n_{i}}, r_{n_{i}}\right)=: V$. Thus, $V$ is an open neighbourhood of $x$ and we can choose some rational $r$ with $0<r<s$ such that $d\left(x_{n_{i}}, x\right)+r<r_{n_{i}}$ for all $i=0, \ldots, m$, especially $B(x, r) \subseteq V$. Now there exists some $y \in \mathbb{Q}^{n}$ such that $\|f(x)-y\|<r$ and still $\partial B(y, s) \subseteq \bigcup_{i=0}^{m} B\left(y_{n_{i}}, r_{n_{i}}\right)$. For this choice of $y, r, n_{0}, \ldots, n_{m}, t:=\frac{s}{r}$ and $W \in C(x, r)$ all conditions (1) to (5) are fulfilled: (1) holds by definition of $W$. (2) holds since by definition $x \in W$ and $f(x) \in B(y, r)$ and thus $(x, f(x)) \in \operatorname{graph}(f) \cap U$. (4) holds by choice of $y$ and $t$ and (3) by choice of $r$. (5) holds since $t r=s<2^{-k}$ by choice of $s$ and definition of $t$. 
This result especially applies to computable normed spaces $X$ and other spaces with connected balls (such as locally convex spaces). The reader might notice that we have not used any specific property of the target space $\mathbb{R}^{n}$ besides the one expressed in Lemma 8.2. We directly obtain the following non-uniform corollary.

Corollary 8.4 Let $X$ be an effectively locally connected computable metric space and let $f: X \rightarrow \mathbb{R}^{n}$ be a continuous function. Then $f$ is computable, if and only if $\operatorname{graph}(f)$ is recursive closed.

Example 5.3 already shows that "effective local connectedness" is not a superfluous condition, neither in the previous result nor in its uniform version. The following proposition shows that in the uniform case the target space $Y=\mathbb{R}^{n}$ cannot be replaced by an arbitrary computable metric space (not even if we restrict ourselves to linear operators). The result is based on the following easy lemma and results from [6].

Lemma 8.5 Let $X, Y$ be computable metric spaces. The "swap map"

$$
S: \mathcal{A}(X \times Y) \rightarrow \mathcal{A}(Y \times X), A \mapsto\{(y, x) \in Y \times X:(x, y) \in A\}
$$

is computable.

The proof follows from the obvious fact that the pointwise "swap function" $s: X \times Y \rightarrow$ $Y \times X,(x, y) \mapsto(y, x)$ operates on centers of balls, i.e.

$$
s B((x, y), r)=B((y, x), r)=B(s(x, y), r)
$$

for all $(x, y) \in X \times Y$ and $r>0$, provided that $X \times Y$ and $Y \times X$ are endowed with the product space structure according to Proposition 3.2.

Proposition 8.6 The mapping graph ${ }^{-1}: \subseteq \mathcal{A}\left(\ell_{2} \times \ell_{2}\right) \rightarrow \mathcal{C}\left(\ell_{2}, \ell_{2}\right)$, defined for all closed subsets $A \subseteq \ell_{2} \times \ell_{2}$ such that $A=\operatorname{graph}(T)$ for some linear bounded and bijective operator $T: \ell_{2} \rightarrow \ell_{2}$ with $\|T\|=1$, is discontinuous.

Proof. Let us assume that graph ${ }^{-1}: \subseteq \mathcal{A}\left(\ell_{2} \times \ell_{2}\right) \rightarrow \mathcal{C}\left(\ell_{2}, \ell_{2}\right)$ would be continuous in the stated sense. By the previous lemma and the weak Graph Theorem 5.1 it follows that the inversion $I: \subseteq \mathcal{C}\left(\ell_{2}, \ell_{2}\right) \rightarrow \mathcal{C}\left(\ell_{2}, \ell_{2}\right), T \mapsto T^{-1}$, restricted to linear bounded and bijective operators $T: \ell_{2} \rightarrow \ell_{2}$ with $\|T\|=1$, would be continuous too, since $I=$ graph $^{-1} \circ S$ ograph. This contradicts Example 17 in [6].

Finally, we want to prove that with the same assumptions on the spaces Theorem 8.3 cannot be strengthened in the way that only negative information on the graph is required in order to determine the function. In the following example we consider $\mathbb{R} \backslash \mathbb{N}$ as a computable metric subspace of $\mathbb{R}$, endowed with the restriction of the Euclidean metric to $X \times X$. 
Example 8.7 Consider $X=\mathbb{R} \backslash \mathbb{N}$ as a computable metric subspace of $\mathbb{R}$ endowed with the restriction of the Euclidean metric. There exists a continuous but non-computable function $f: X \rightarrow \mathbb{R}$ such that $\operatorname{graph}(f) \subseteq X \times \mathbb{R}$ is strongly co-r.e. closed.

Proof. Let $a: \mathbb{N} \rightarrow \mathbb{N}$ be some computable and injective enumeration of a non-recursive set $K=\operatorname{range}(a)$. Define $f: \mathbb{R} \backslash \mathbb{N} \rightarrow \mathbb{R}$ by

$$
f(x):=\left\{\begin{array}{l}
n+1 \text { if }(\exists n, k) x \in(k, k+1) \text { and } a(n)=k \\
0 \quad \text { else }
\end{array}\right.
$$

for all $x \in \mathbb{R} \backslash \mathbb{N}$. Then $f$ is continuous but non-computable since $K=\operatorname{range}(a)$ is not co-r.e. and

$$
k \notin K \Longleftrightarrow f\left(k+\frac{1}{2}\right)<1 .
$$

Moreover, graph $(f)$ is strongly co-r.e. closed since $a$ is computable and

$$
\begin{aligned}
& ([c, d] \times[r, s]) \cap \operatorname{range}(f)=\emptyset \\
\Longleftrightarrow & (\exists k)((k<c \leq d<k+1 \text { and }(\forall n+1 \in[r, s])(a(n) \neq k)) \\
& \text { and }(0 \in[r, s] \Longrightarrow(\exists n)(a(n)=k)))
\end{aligned}
$$

for all $c, d, r, s \in \mathbb{Q}$ such that $r \leq s$ and there exists some $k$ with $k<c \leq d<k+1$. The general case of those $c \leq d$ which are not subject to the previous inequality can easily be reduced to the presented special case.

\section{Pathwise connected source spaces}

In this section we will discuss another connectedness property of the source space which guarantees that the uniform inverse direction of the Graph Theorem becomes computable, even if essentially only negative information on the graph is available. The only positive information which we will require is a single point $(a, b) \in \operatorname{graph}(f)$.

We will call a computable metric space $X$ effectively pathwise connected, if for all points $x, y \in X$ we can effectively find some continuous function $p:[0,1] \rightarrow X$ such that $p(0)=x$ and $p(1)=y$. More precisely:

Definition 9.1 (Effective pathwise connectedness) A computable metric space $X$ is called effectively pathwise connected, if there exists a computable multi-valued function $P: X \times X \rightrightarrows \mathcal{C}([0,1], X)$ such that for all $x, y \in X$ and $p \in P(x, y)$ it holds that $p(0)=x$ and $p(1)=y$. 
It is easy to see that the Euclidean space $\mathbb{R}^{n}$ and computable normed spaces $X$ are effectively pathwise connected (a path $p:[0,1] \rightarrow X$ can simply be obtained by $p(r):=$ $x+r(y-x)$ in these cases). Now we are prepared to prove the following theorem. The proof is a modified version of the proof of Theorem 8.3.

Theorem 9.2 (Pathwise connected Graph Theorem) Let $X$ be an effectively pathwise connected computable metric space and let $n \geq 1$ be a natural number. The mapping

$$
F: \subseteq \mathcal{A}_{>}\left(X \times \mathbb{R}^{n}\right) \times X \times \mathbb{R}^{n} \rightarrow \mathcal{C}\left(X, \mathbb{R}^{n}\right),(A, a, b) \mapsto \operatorname{graph}^{-1}(A)
$$

is computable, which is defined for all $(A, a, b)$ such that $A=\operatorname{graph}(f)$ and $f(a)=b$ for some $f \in \mathcal{C}\left(X, \mathbb{R}^{n}\right)$.

Proof. We consider the computable metric space $(X, d, \alpha)$. Let $f: X \rightarrow \mathbb{R}^{n}$ be a continuous function and let $(a, b) \in X \times \mathbb{R}^{n}$ with $f(a)=b$. Furthermore, let $x \in X$ and a precision $k \in \mathbb{N}$ be given. We prove that we can effectively evaluate $f(x)$ up to precision $2^{-k}$ which implies by type conversion the desired result. We can assume that graph $(f)$ is given as point graph $(f) \in \mathcal{A}_{>}\left(X \times \mathbb{R}^{n}\right)$ and thus we can find points $\left(x_{i}, y_{i}\right) \in \operatorname{range}(\alpha) \times \mathbb{Q}^{n}$ and rational numbers $r_{i}>0$ such that

$$
\operatorname{graph}(f)^{c}=\left(X \times \mathbb{R}^{n}\right) \backslash \operatorname{graph}(f)=\bigcup_{i=0}^{\infty}\left(B\left(x_{i}, r_{i}\right) \times B\left(y_{i}, r_{i}\right)\right) .
$$

Since $X$ is effectively pathwise connected, we can effectively find a continuous function $p:[0,1] \rightarrow X$ such that $p(0)=a$ and $p(1)=x$. The strategy of the proof is to follow the graph along the path $p$ from $a$ to $x$ in order to evaluate $f$ at $x$. Therefore, we systematically search for values $m \in \mathbb{N}$, a partition $0=a_{0}<a_{1}<a_{2}<\ldots<a_{m}=1$ with $a_{j} \in \mathbb{Q}$ and we guess possible function values $b_{0}:=b, b_{j+1} \in B\left(b_{j}, 2^{-k}\right) \cap \mathbb{Q}^{n}$ for $j=0, \ldots, m-1$ such that

$$
p\left[a_{j}, a_{j+1}\right] \times \partial B\left(b_{j}, 2^{-k}\right) \subseteq \bigcup_{i=0}^{\infty}\left(B\left(x_{i}, r_{i}\right) \times B\left(y_{i}, r_{i}\right)\right) .
$$

We first mention that the inclusion in Equation (1) is r.e. in all parameters, by the computable Heine-Borel Theorem (cf. Theorems 4.6 and 4.10 in [8]). This follows from Lemma 8.2 together with the fact that the mapping $C([0,1], X) \times \mathcal{K}[0,1] \rightarrow \mathcal{K}(X),(p, A) \mapsto$ $p(A)$ is computable (see Theorem 3.3 in [19] and Theorem 4.12 in [7]).

Now we claim that always appropriate values $m, a_{j}, b_{j}$ exist such that Equation (1) is fulfilled and we claim that $\|f(x)-y\|<2^{-k}$ holds for $y:=b_{m}$ in this case.

Thus, let us first assume that Equation (1) holds. Since $f p:[0,1] \rightarrow \mathbb{R}^{n}$ is continuous, it follows that $\operatorname{graph}(f p)$ is connected and since $f p\left(a_{0}\right)=f p(0)=f(a)=b=b_{0}$, it follows

$$
\operatorname{graph}(f p) \subseteq \bigcup_{j=0}^{m-1}\left(\left[a_{j}, a_{j+1}\right] \times B\left(b_{j}, 2^{-k}\right)\right)
$$


In particular, $f(x)=f p(1)=f p\left(a_{m}\right) \in B\left(b_{m}, 2^{-k}\right)$ and thus $\|f(x)-y\|<2^{-k}$ as we have claimed.

Now we still have to prove that there always exist appropriate values $m, a_{j}, b_{j}$ such that Equation (1) is fulfilled. Since $f p:[0,1] \rightarrow \mathbb{R}^{n}$ is continuous and $[0,1]$ is compact, it follows that $f p$ is even uniformly continuous and there exists some value $l \in \mathbb{N}$ such that $f p\left[a_{j}, a_{j+1}\right] \subseteq B\left(f p\left(a_{j}\right), 2^{-k-1}\right)$ for all $j=0, \ldots, m-1$ with $m:=2^{l}$ and $a_{j}:=j \cdot 2^{-l}$. Thus, if we choose $b_{j} \in \mathbb{Q}^{n}$ such that $\left\|f p\left(a_{j}\right)-b_{j}\right\| \leq 2^{-k-1}$, then Equation (1) is fulfilled.

Since in case of complete spaces $X$ positive information on the graph allows to find some $x$ and $y$ with $f(x)=y$ (which follows from Proposition 4.4), we obtain the following corollary.

Corollary 9.3 Let $X$ be an effectively pathwise connected and complete computable metric space. The mapping graph $: \mathcal{C}\left(X, \mathbb{R}^{n}\right) \rightarrow \mathcal{A}\left(X \times \mathbb{R}^{n}\right), f \mapsto \operatorname{graph}(f)$ as well as its inverse graph ${ }^{-1}: \subseteq \mathcal{A}\left(X \times \mathbb{R}^{n}\right) \rightarrow \mathcal{C}\left(X, \mathbb{R}^{n}\right)$ are computable.

We do not know whether completeness is a superfluous property in this case. In the nonuniform case we have learned by Proposition 7.2 that a continuous function $f: X \rightarrow \mathbb{R}^{n}$ with a co-r.e. graph always maps computable inputs to computable outputs. Therefore we obtain the following corollary of the previous theorem.

Corollary 9.4 Let $X$ be an effectively pathwise connected computable metric space and let $f: X \rightarrow \mathbb{R}^{n}$ be a continuous function. Then $f$ is computable, if and only if graph $(f)$ is recursive closed, if and only if graph $(f)$ is co-r.e. closed.

Moreover, we can easily conclude a linear version of the previous theorem (where we consider only negative information on the graph) since in the linear case $f(0)=0$ and thus the additional input information (a pair from the graph of $f$ ) is automatically available. Here, a computable normed space is a computable metric space with an additional vector space structure such that the algebraic operations are computable (see e.g. [6] for details).

Corollary 9.5 Let $X$ be a computable normed space and let $n \geq 1$ be a natural number. The mapping graph $: \subseteq \mathcal{C}\left(X, \mathbb{R}^{n}\right) \rightarrow \mathcal{A}_{>}\left(X \times \mathbb{R}^{n}\right), f \mapsto \operatorname{graph}(f)$, defined for linear mappings $f: X \rightarrow \mathbb{R}^{n}$, as well as its partial inverse graph ${ }^{-1}: \subseteq \mathcal{A}_{>}\left(X \times \mathbb{R}^{n}\right) \rightarrow \mathcal{C}\left(X, \mathbb{R}^{n}\right)$ are computable.

\section{Conclusions}

The following table gives a survey of our results. We assume that $X, Y$ are computable metric spaces and $f: X \rightarrow Y$ is a continuous function. Each double row lists a certain version of the Graph Theorem (where the first row of any double row contains a nonuniform version and the second row the corresponding uniform version). The first double row treats the easy direction of the Graph Theorem, the remaining double rows of the 
table consider the problematic direction and they are split into three cases where positive, negative and both types of information on graphs are considered (in reverse order). The top of the columns lists different additional conditions on the spaces $X$ and $Y$ (and on the functions $f$ in the second half which treats the linear case). Any symbol "+" indicates that the statement of the corresponding row holds in general under the assumptions of the corresponding column. Any "-_" indicates that there are counterexamples of spaces (and functions) which fulfill the assumptions of the corresponding column but the statement of the corresponding row does not hold in case of the counterexample (in the uniform case it typically indicates that the corresponding mapping is not even continuous). Finally, the bottom of the columns contains examples of functionalities $X \rightarrow Y$ which simultaneously meet all indicated properties of the corresponding column.

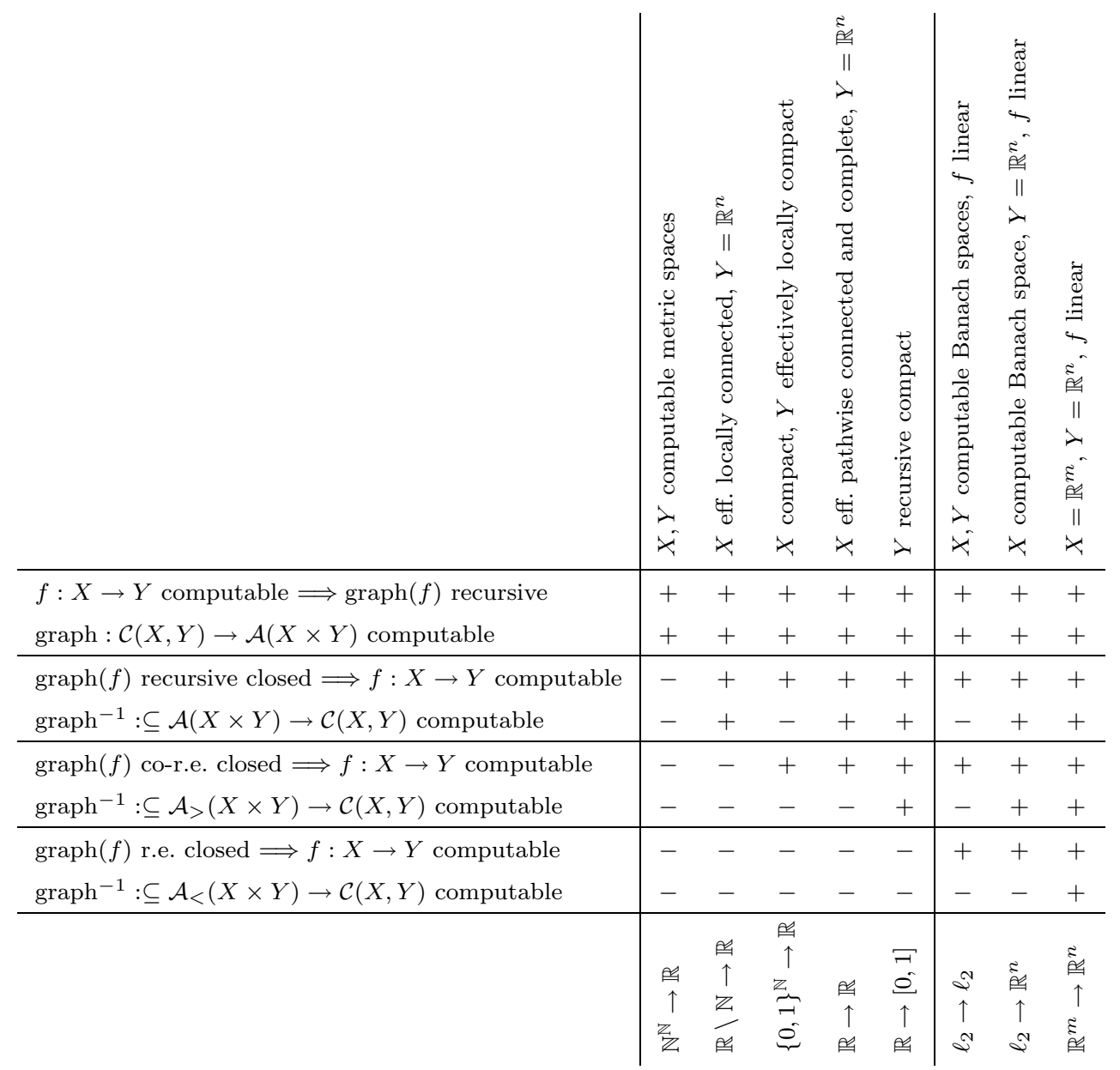

Fig. 5. Survey of different versions of the Graph Theorem.

The results of the first double row follow directly from Theorem 5.1, the weak version of the Graph Theorem. The positive results in the second column follow from Theorem 8.3, the Graph Theorem for locally connected source spaces. The additional positive results in the third column follow from Corollary 7.3, the Graph Theorem for compact source spaces and locally compact target spaces. The positive results in the fourth column follow from Corollaries 9.3 and 9.4, the Graph Theorem for pathwise connected source spaces. The positive results in the fifth column follow from Theorem 6.2, the Graph Theorem 
for compact target spaces. All positive results in the first row of the second, third and fourth double row concerning the linear case follow from the effective version of the Closed Graph Theorem [2]. The remaining positive results in the second double row are implied by the corresponding positive results of the second column. The remaining positive results in the third double row follow from Corollary 9.5. The remaining positive result of the fourth double row is a consequence of Theorem 4.7 in [5]. All negative results in the first column follow from Example 5.3 for the space $X=\mathbb{N}^{\mathbb{N}}$ and Proposition 5.2. The negative results in the second column of the third double row follow from Example 8.7 for the space $X=\mathbb{R} \backslash \mathbb{N}$. The negative results in the third column, second and third double row, follow from Proposition 5.2 for the space $X=\{0,1\}^{\mathbb{N}}$. The negative results in the second, third, fourth and fifth column of the fourth double row follow from Example 6.6 for the spaces $X=Y=[0,1]$ or $X=Y=\mathbb{R}$. This example can easily be modified in order to obtain the negative results concerning the third column for the space $X=\{0,1\}^{\mathbb{N}}$. The remaining negative result in the fourth column is a consequence of Proposition 6.8 for the spaces $X=Y=\mathbb{R}$. The negative results in the sixth column are consequences of Proposition 8.6 for the spaces $X=Y=\ell_{2}$. The negative result in the seventh column is a consequence of Theorem 4.5 in [5]. It should be mentioned that completeness in the fourth column has only been used for the positive result of the third row.

Of course the table leaves open a lot of further questions. We just formulate one example:

Open Problem. Is there an effectively pathwise connected computable metric space $X$ such that graph ${ }^{-1}: \subseteq \mathcal{A}\left(X \times \mathbb{R}^{n}\right) \rightarrow \mathcal{C}\left(X, \mathbb{R}^{n}\right)$ is not computable?

By the known results it follows that such a space $X$ can neither be effectively locally connected nor complete. The space $X=(\mathbb{R} \times\{0\}) \cup(\mathbb{Q} \times \mathbb{R})$, endowed with the subtopology of the Euclidean topology is an example of an effectively pathwise connected computable metric space which is neither locally connected nor complete and thus a potential candidate.

One interesting consequence of the Graph Theorem is that it allows certain conclusions with respect to the inversion problem: spaces $X, Y$ such that the mapping from $\operatorname{graph}(f)$ to $f: X \rightarrow Y$ is computable also have the property that the mapping from any homeomorphism $g: Y \rightarrow X$ to its inverse $g^{-1}: X \rightarrow Y$ is computable (in the uniform case, as well as in the non-uniform case). This follows since information on the graph is symmetric (this is made precise by Lemma 8.5). Thus, in all those cases where the second double row in Figure 5 carries a plus, it follows that inversion is effective. However, the negative results cannot be transfered that easily (since it might happen that inversion can be established without using the graph as intermediate step). This interesting problem deserves further investigations. 


\section{References}

[1] S. Banach and S. Mazur, Sur les fonctions calculables, Ann. Soc. Pol. de Math. 16 (1937) 223.

[2] V. Brattka, Computability of Banach space principles, Informatik Berichte 286, FernUniversität Hagen, Fachbereich Informatik, Hagen, June 2001.

[3] V. Brattka, Random numbers and an incomplete immune recursive set, in: P. Widmayer, F. Triguero, R. Morales, M. Hennessy, S. Eidenbenz, and R. Conejo (eds.), Automata, Languages and Programming, vol. 2380 of Lect. Not. Comp. Sci., Springer, Berlin 2002, 950-961.

[4] V. Brattka, Computability over topological structures, in: S. B. Cooper and S. S. Goncharov (eds.), Computability and Models, Kluwer Academic Publishers, New York 2003, 93-136.

[5] V. Brattka, Effective representations of the space of linear bounded operators, Applied General Topology 4 (2003) 115-131.

[6] V. Brattka, The inversion problem for computable linear operators, in: H. Alt and M. Habib (eds.), STACS 2003, vol. 2607 of Lect. Not. Comp. Sci., Springer, Berlin 2003, 391-402.

[7] V. Brattka and G. Presser, Computability on subsets of metric spaces, Theoret. Comp. Sci. (2003) (to appear).

[8] V. Brattka and K. Weihrauch, Computability on subsets of Euclidean space I: Closed and compact subsets, Theoret. Comp. Sci. 219 (1999) 65-93.

[9] D. Cenzer and J. B. Remmel, Effectively closed sets and graphs of computable real functions, Theoret. Comp. Sci. 284 (2002) 279-318.

[10] C. Ceola and P. B. Lecomte, Computability of a map and decidability of its graph in the model of Blum, Shub and Smale, Theoret. Comp. Sci. 194 (1998) 219-223.

[11] A. Grzegorczyk, On the definitions of computable real continuous functions, Fund. Math. 44 (1957) 61-71.

[12] K.-I. Ko, Complexity Theory of Real Functions, Birkhäuser, Boston 1991.

[13] D. Lacombe, Extension de la notion de fonction récursive aux fonctions d'une ou plusieurs variables réelles I-III, Comptes Rendus 240,241 (1955) 2478-2480,13-14,151-153.

[14] J. S. Miller, Effectiveness for embedded spheres and balls, in: V. Brattka, M. Schröder, and K. Weihrauch (eds.), CCA 2002 Computability and Complexity in Analysis, vol. 66 of Electronic Notes in Theoretical Computer Science, Elsevier, Amsterdam 2002.

[15] P. Odifreddi, Classical Recursion Theory, North-Holland, Amsterdam 1989.

[16] M. B. Pour-El and J. I. Richards, Computability in Analysis and Physics, Springer, Berlin 1989.

[17] A. M. Turing, On computable numbers, with an application to the "Entscheidungsproblem", Proc. London Math. Soc. 42 (1936) 230-265. 
[18] K. Weihrauch, Computable Analysis, Springer, Berlin 2000.

[19] K. Weihrauch, Computational complexity on computable metric spaces, Math. Log. Quart. 49 (2003) 3-21.

[20] M. Yasugi, T. Mori, and Y. Tsujii, Effective properties of sets and functions in metric spaces with computability structure, Theoret. Comp. Sci. 219 (1999) 467-486.

[21] Q. Zhou, Computable real-valued functions on recursive open and closed subsets of Euclidean space, Math. Log. Quart. 42 (1996) 379-409. 